\title{
Defect-Triggered Phase Transition in Cesium Lead Halide Perovskite Nanocrystals
}

\author{
Ju-Ping Ma, ${ }^{\dagger, \# \odot ~ J u n ~ Y i n, ~}{ }^{\dagger, \# \odot ~ Y a-M e n g ~ C h e n, ~}{ }^{\dagger, \#}$ Qing Zhao, ${ }^{\S, \#}$ Yang Zhou, ${ }^{\dagger}$ Hong Li, ${ }^{\dagger}$ \\ Yoshihiro Kuroiwa, ${ }^{\S}$ Chikako Moriyoshi, ${ }^{\S}$ Zhi-Yong Li, ${ }^{\dagger}$ Osman M. Bakr, ${ }^{\ddagger}$ Omar F. Mohammed, ${ }^{*}, \ddagger$ \\ and Hong-Tao Sun $*, \dagger, \|_{\odot}$
}
${ }^{\dagger}$ College of Chemistry, Chemical Engineering and Materials Science, Soochow University, Suzhou, Jiangsu 215123, China
${ }^{\ddagger}$ Division of Physical Sciences and Engineering, King Abdullah University of Science and Technology (KAUST), Thuwal 23955-6900, Kingdom of Saudi Arabia
${ }^{\S}$ Department of Physical Science, Hiroshima University, Higashihiroshima, Hiroshima 739-8526, Japan
"State Key Laboratory of Luminescence and Applications, Changchun Institute of Optics, Fine Mechanics and Physics, Chinese Academy of Sciences, Changchun, 130033, China

\section{Supporting Information}

\begin{abstract}
All-inorganic perovskite nanocrystals (NCs) have emerged as a class of low-cost and high-efficiency light-emitting materials. However, the structure and phase transition behavior of these NCs remain poorly understood. Here, we provide unambiguous evidence that all these properties are associated with structural defects in NCs. Using $\mathrm{CsPbl}_{3} \mathrm{NCs}$ as a model system, we find that the cubic subdomains in highly defective NCs gradually convert to the orthorhombic upon cooling room-temperature quasi-cubic NCs, whereas high-quality cousins, with mixed cubic and orthorhombic subdomains at room temperature, exhibit a significant resistance for such a phase transition. Car-Parrinello molecular dynamics simulations unveil the important role of point defects in triggering a stepwise, reversible phase transition of $\mathrm{CsPbCl}_{3}$ NCs. Importantly, we show that the defect-triggered phase transition also exists in other all-inorganic perovskite NCs. Our findings provide new insight into the structure and phase transition of $\mathrm{CsPbX}_{3} \mathrm{NCs}$ and highlight the important role of defects in impacting these properties.
\end{abstract}

$\mathrm{L}$ ead halide perovskites such as methylammonium lead iodide $\left(\mathrm{CH}_{3} \mathrm{NH}_{3} \mathrm{PbI}_{3}\right.$ or $\left.\mathrm{MAPbI}_{3}\right)$ have received wideranging attention owing to their outstanding optoelectronic properties, which lend them to application in highefficiency solar cells, light-emitting devices, and detectors. ${ }^{1-6}$ The optoelectronic properties of lead halide perovskites are typically tailored by modifying the chemical compositions (e.g., partial replacement of cationic or anionic ions by other elements that can retain the perovskite structure). ${ }^{7-12}$ Besides, tuning temperature, one of the most accessible strategies, has been gradually recognized as a useful knob for increasing the tunability of perovskite's properties by virtue of the phase transition. ${ }^{13-20}$ For instance, $\mathrm{MAPbI}_{3}$ is cubic at temperatures above $330 \mathrm{~K}$, tetragonal between $330 \mathrm{~K}$ and $\sim 160 \mathrm{~K}$, and finally transforms to a low-symmetry orthorhombic crystal structure below $\sim 160 \mathrm{~K}^{13}$ Interestingly, recent studies have shown that the temperature of the tetragonal-to-orthorhombic phase transition of $\mathrm{MAPbI}_{3}$ is intimately associated with structural defects, ${ }^{19}$ which leads to the coexistence of tetragonal and orthorhombic phases at low temperatures.
The great benefit of dual-phase coexistence lies in drastically enhanced emission quantum yields, ${ }^{21}$ which result in the first demonstration of continuous-wave lead halide perovskite lasers. $^{22}$ Interestingly, the phase transition of defect-bearing $\mathrm{MAPbI}_{3}$ is not totally reversible at around the phase transition temperature, as reflected by the temperature hysteresis. ${ }^{19}$

To overcome the instability issue of hybrid perovskites, recent efforts have been devoted to cesium lead halide perovskite $\left(\mathrm{CsPbX}_{3}, \mathrm{X}=\right.$ halide ions) nanocrystals (NCs) due to their good stability, unique optical versatility, high luminescence quantum yields, and facile synthesis. ${ }^{23-33}$ However, in contrast to well-studied bulk $\mathrm{CsPbX}_{3}{ }^{34-39}$ the phase transition behavior and photophysical properties of $\mathrm{CsPbX}_{3} \mathrm{NCs}$ have yet to be disserted fully. Until now, only a few studies of the phase transition have been conducted on

Received: April 26, 2019

Accepted: June 14, 2019

Published: June 14, 2019 

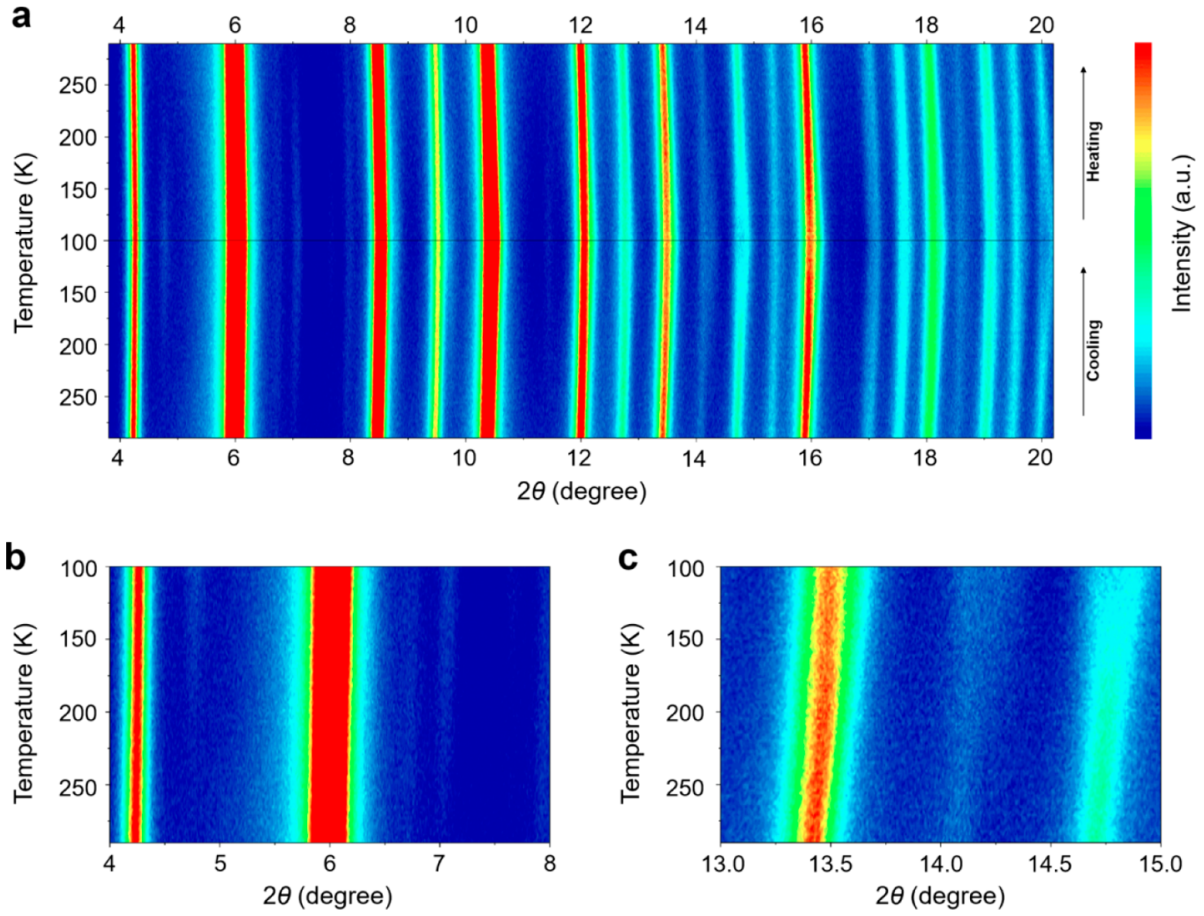

Figure 1. (a) Temperature-dependent XRD patterns of highly defective $\mathrm{CsPbCl}_{3} \mathrm{NCs}$ during the cooling and heating cycle; the NCs were first cooled from 290 to $100 \mathrm{~K}$ and then heated from 100 to $290 \mathrm{~K}$ with a rate of $10 \mathrm{~K} / \mathrm{min}$. (b, c) Temperature-dependent XRD patterns of NCs in the ranges of $4^{\circ}-8^{\circ}(\mathrm{b})$ and $13^{\circ}-15^{\circ}$ (c) upon cooling from 290 to $100 \mathrm{~K}$.

such material systems. ${ }^{40-43}$ For instance, Bertolotti and coworkers investigated the structures of $\mathrm{CsPbX}_{3} \mathrm{NCs}$ and suggested that the local structure of these NCs always exhibits orthorhombic tilting of $\mathrm{PbX}_{6}$ octahedra within locally ordered subdomains that are hinged through a two-/three-dimensional network of twin boundaries. ${ }^{40}$ However, no obvious phase transition for $\mathrm{CsPbCl}_{3} \mathrm{NCs}$ has been observed at low temperatures, as signified by the absence of superstructure diffraction peaks of the orthorhombic phase. Furthermore, the full details of the phase transition and the nature of the mechanism for the formation of multi-subdomains in individual $\mathrm{CsPbX}_{3} \mathrm{NCs}$ remain essentially unexplored. Previous works have revealed that $\mathrm{CsPbX}_{3} \mathrm{NCs}$, similar to their hybrid counterparts, are highly defective. ${ }^{23,44,45}$ Nevertheless, to date, there exists no convincing prior identification of how defects influence the phase transition of $\mathrm{CsPbX}_{3} \mathrm{NCs}$. We took the view that understanding these challenging scientific issues could provide insight into unconventional optoelectronic properties observed in $\mathrm{CsPbX}_{3} \mathrm{NCs}^{46,47}$

Here, we present an in-depth investigation of the phase transition of $\mathrm{CsPbX}_{3} \mathrm{NCs}$ across a wide temperature range by a combination of experimental characterizations and molecular dynamics simulations. We discover that the reversible phase transition behavior of $\mathrm{CsPCl}_{3} \mathrm{NCs}$ is intimately linked with structural defects. Highly defective $\mathrm{CsPbCl}_{3} \mathrm{NCs}$ are quasicubic at room temperature (RT), in which the cubic subdomains gradually convert to the orthorhombic phase upon cooling. By contrast, high-quality $\mathrm{CsPbCl}_{3} \mathrm{NCs}$ show a greatly retarded phase transition from $\mathrm{RT}$ mixed cubic and orthorhombic subdomains. Molecular dynamics simulations demonstrate that point defects such as chloride vacancies play a pivotal role in triggering the phase transition of $\mathrm{CsPCl}_{3}$ NCs. We also discuss the generality of our findings in other $\mathrm{CsPbX}_{3}$ NCs. Our work offers new insight into the structure and phase transition of $\mathrm{CsPbX}_{3} \mathrm{NCs}$ and emphasizes the important role of defects in impacting their structures.

The $\mathrm{CsPbCl}_{3} \quad \mathrm{NCs}$ were synthesized by a hot-injection method as first reported by Protesescu and coworkers. ${ }^{23}$ The average edge length of $\mathrm{CsPbCl}_{3} \mathrm{NCs}$ is $10.9 \mathrm{~nm}$ (Figure S1). These NCs show an exciton absorption band at $397 \mathrm{~nm}$ and band-edge emission at $405 \mathrm{~nm}$ with a photoluminescence quantum yield (PLQY) of $1.2 \%$, consistent with previous results. $^{23,48}$ Such a low PLQY indicates a highly defective lattice that contains chlorine and/or chlorine-related defects. $^{44,48}$ To avoid any possible adverse effect of water molecules on the structure, the as-synthesized NCs were stored in a $\mathrm{N}_{2}$-filled glovebox for the following measurements. The preliminary structural characterization based on laboratory $\mathrm{X}$ ray diffraction (XRD) suggests the formation of cubic $\mathrm{CsPCl}_{3}$ at RT (Figure S2). We then performed the temperaturedependent synchrotron XRD measurements at the BL02B2 beamline of SPring- 8 , using a MYTHEN detector system, ${ }^{49}$ to examine the phase transition behavior. The NCs sealed in a capillary were first cooled from 290 to $100 \mathrm{~K}$ and then heated to $290 \mathrm{~K}$, with a same cooling/heating rate of $10 \mathrm{~K} / \mathrm{min}$. As illustrated in Figure 1a, it is apparent that the phase transition of $\mathrm{CsPCl}_{3} \mathrm{NCs}$ is reversible, indicating that, different from some hybrid cousins, ${ }^{19}$ the long-range order of the $\mathrm{CsPbl}_{3}$ $\mathrm{NCs}$ is well retained after undergoing phase transition, although they are defective. We stress that the reversible phase transition behavior of $\mathrm{CsPbCl}_{3} \mathrm{NCs}$ has not been reported so far. We note, upon cooling, a superstructure peak at $7.07^{\circ}$ develops at $\sim 270 \mathrm{~K}$ (Figure 1b), which can be assigned to the orthorhombic $\mathrm{CsPbCl}_{3}$. Further decreasing the temperature leads to the appearance of more superstructure peaks at $\sim 4.77^{\circ}, 6.76^{\circ}, 7.67^{\circ}$, and $7.99^{\circ}$. Interestingly, we find that as the temperature decreases, the peak corresponding to the (222) plane of the RT cubic $\mathrm{CsPCl}_{3}$ is gradually 

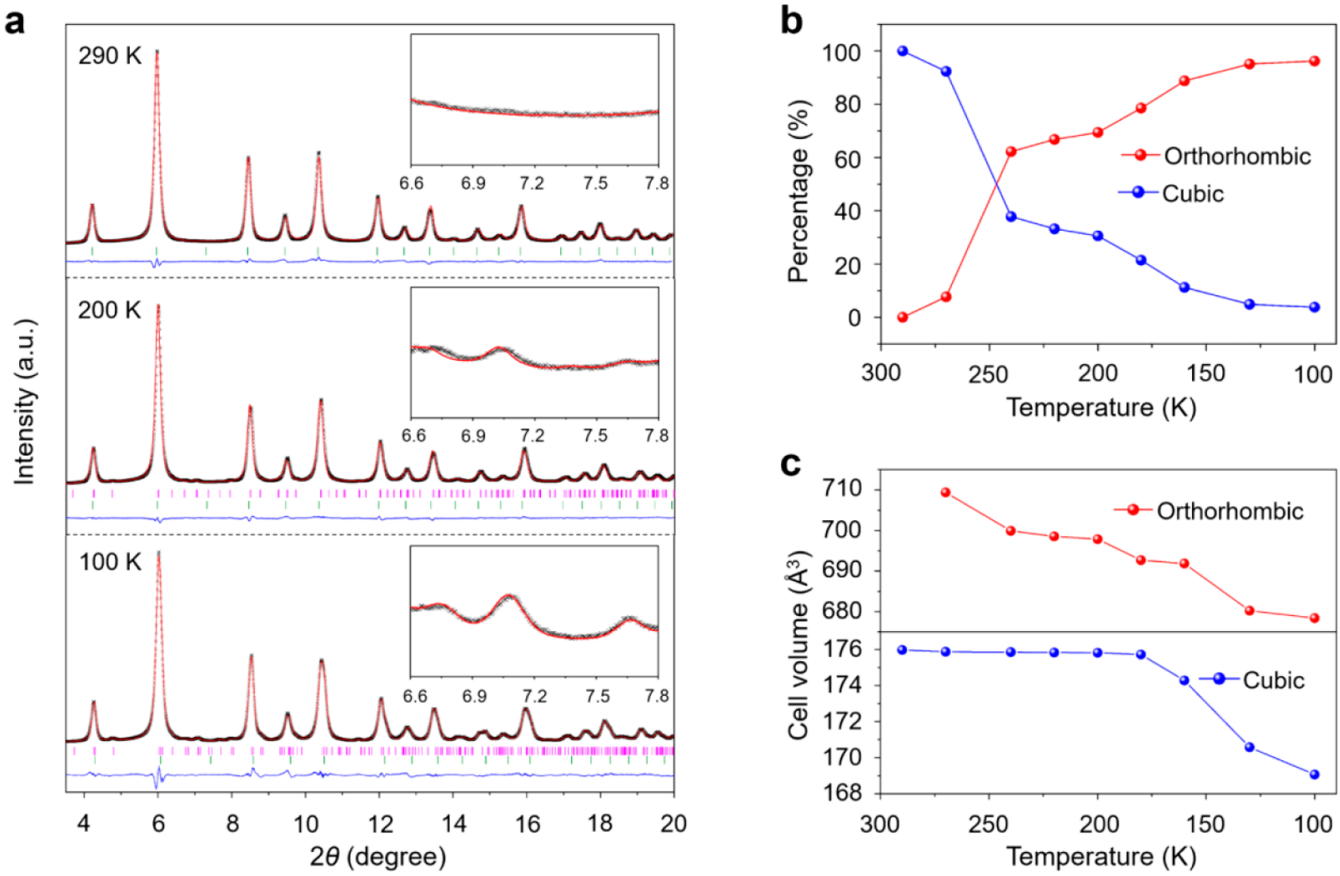

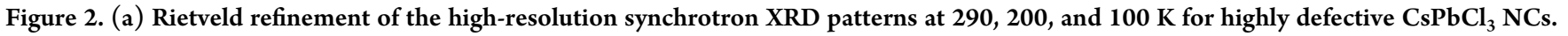
The insets show the enlarged patterns corresponding to the peaks of the orthorhombic phase. The red lines and overlying crosses show the calculated and observed intensities, respectively. The pink and green lines indicate the positions of the calculated Bragg reflections of orthorhombic and cubic $\mathrm{CsPbCl}_{3}$, respectively. The difference between the observed and calculated profiles is plotted in blue. (b, c) Percentages (b) and cell volumes (c) of the cubic and orthorhombic phases in highly defective $\mathrm{CsPbCl}_{3} \mathrm{NCs}_{\text {at }}$ different temperatures.

broadened, accompanied by weakened intensity and notable peak splitting at $100 \mathrm{~K}$ (Figure $1 \mathrm{c}$ and Figure S3). These observations unambiguously provide fingerprints of $\left[\mathrm{PbCl}_{6}\right]$ octahedral tilts taking place at $270 \mathrm{~K}$ and of a stepwise phase transition occurring over an extremely broad temperature window (100-270 K).

To gain deep insight into this unconventional, stepwise phase transition, we next took high-resolution synchrotron XRD patterns of $\mathrm{CsPbCl}_{3} \mathrm{NCs}$ at different temperatures, which have been used for Rietveld refinement. Three representative synchrotron XRD spectra are shown in Figure 2a. All diffraction peaks of $\mathrm{CsPbCl}_{3} \mathrm{NCs}$ at $290 \mathrm{~K}$ match the Bragg positions of the cubic $\mathrm{CsPCl}_{3}$, and no impurities exist in the product. Assuming a cubic lattice, Rietveld refinement of the data can be performed quite adequately (Figure 2a and Table S1). In the refinement, the isotropic atomic displacement parameters (ADPs), not the anisotropic ADPs, which were considered as diffraction data from nanosized materials cannot provide robust information. ${ }^{40}$ Owing to the appearance of superstructure peaks that can be assigned to the orthorhombic $\mathrm{CsPbCl}_{3}$ for the pattern at $200 \mathrm{~K}$, we refined this data using a two-phase model because merely refining it using an orthorhombic model shows notable deviation from the experimental data (Figure S4). We find that the weight ratio of the orthorhombic phase in NCs is $69.4 \%$ (Figure 2a and Table S2). Compared with this, refinement of the twophase model against the data taken at $100 \mathrm{~K}$ gives a ratio of $96.2 \%$, signifying that the dominant phase is orthorhombic (Figure 2a and Table S3). We also refined the high-resolution patterns taken at other temperatures, from which we obtain the change in the ratio of the orthorhombic phase in NCs (Figure $2 b$, Figures S5-S10 and Tables S4-S9). Notably, the amount of the orthorhombic phase gradually increases from $7.7 \%$ at $270 \mathrm{~K}$ to $96.2 \%$ at $100 \mathrm{~K}$. As expected, both the lattices of the cubic and orthorhombic phases contract upon decreasing the temperature (Figure 2c). The volume of the unit cell for the cubic phase decreases from $175.980(4) \AA^{3}$ at $290 \mathrm{~K}$ to $169.07(19) \AA^{3}$ at $100 \mathrm{~K}$, while that of the orthorhombic decreases from $709.4(5) \AA^{3}$ at $270 \mathrm{~K}$ to $678.40(5) \AA^{3}$ at $100 \mathrm{~K}$.

To examine the influence of defects on the phase transition behavior, we next prepared high-quality $\mathrm{CsPbCl}_{3} \mathrm{NCs}$ by a modified hot-injection method. We used benzoyl chloride as an additional chloride source to create Cl-rich growth conditions, ${ }^{32}$ which favors the suppression of the formation of $\mathrm{Cl}$ vacancies as we previously revealed. ${ }^{44}$ The resulting NCs, with an edge length of $10.1 \mathrm{~nm}$, display similar absorption and emission bands to their highly defective analogues but exhibit a high PLQY of $78.8 \%$ (Figure S11). Previous work revealed that, different from $\mathrm{Pb}$ and $\mathrm{Cs}$ vacancies, $\mathrm{Cl}$ vacancies can introduce deep in-gap defect levels, which function as charge carrier traps and result in low-efficiency band-edge emission. ${ }^{44}$ We thus conclude that the concentration of $\mathrm{Cl}$ vacancies and/ or Cl-vacancy-related defects in high-quality $\mathrm{CsPbCl}_{3} \mathrm{NCs}$ should be much smaller than that in highly defective cousins. As shown in Figure $3 \mathrm{a}$, these high-quality NCs also demonstrate reversible phase transition in the range of 100$290 \mathrm{~K}$. However, careful inspection of the high-resolution synchrotron XRD patterns suggests that the structure and phase transition behavior of these NCs are different from those of highly defective cousins. Notably, comparison of the RT diffraction patterns of these two kinds of NCs shows that highquality NCs exhibit relatively broad, asymmetric peaks (Figure S12), implying the intrinsic difference in their structures. We further attempt to refine the experimental data using the models of different polymorphs, and the results suggest that the high-quality NCs consist of mixed phases of cubic and orthorhombic at RT (Figure S13). We then carried out Rietveld refinement of the synchrotron XRD data at different 

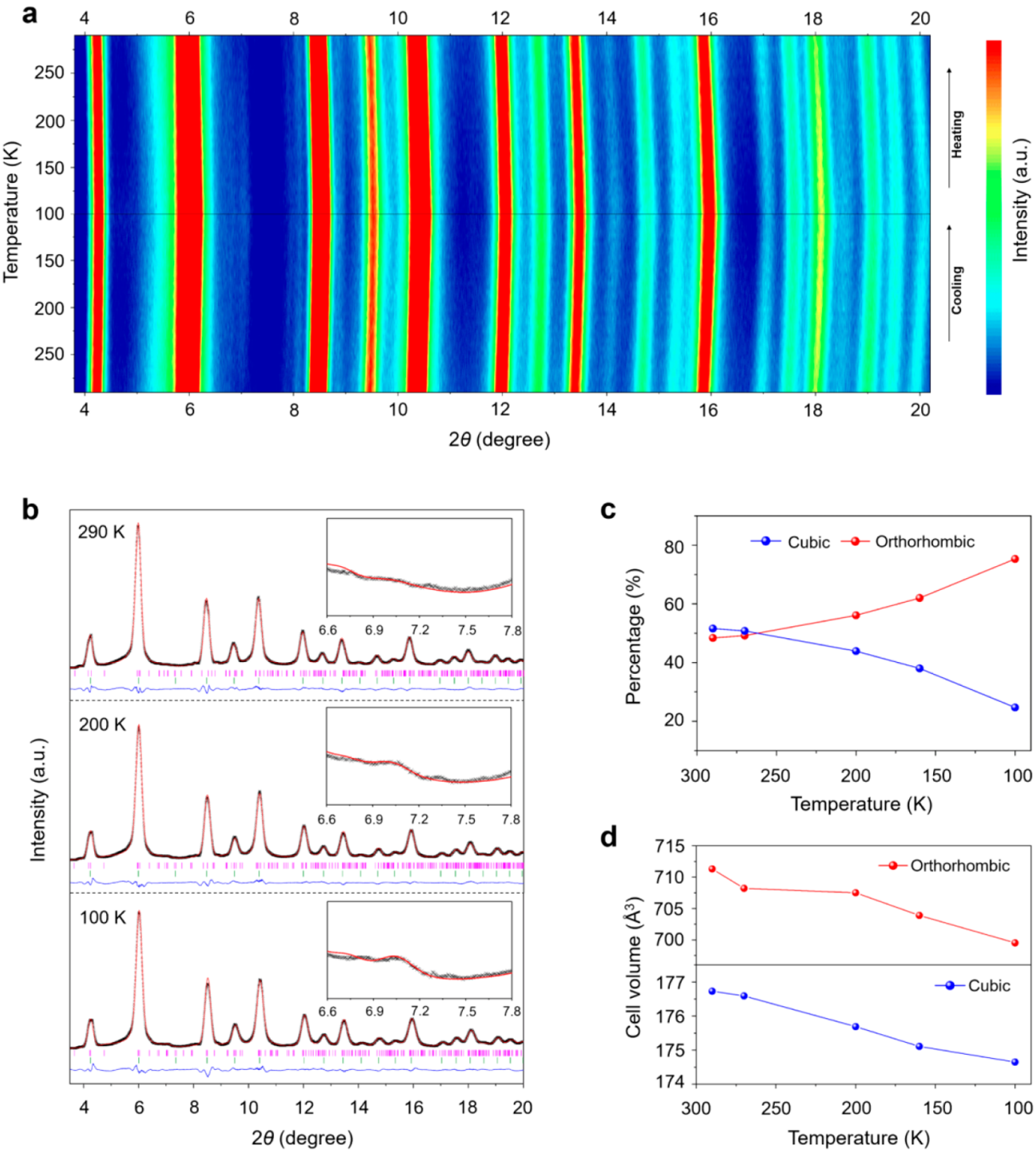

Figure 3. (a) Temperature-dependent XRD patterns of high-quality $\mathrm{CsPbCl}_{3} \mathrm{NCs}$ during the cooling and heating cycle; the NCs were first cooled from 290 to $100 \mathrm{~K}$ and then heated from 100 to $290 \mathrm{~K}$ with a rate of $10 \mathrm{~K} / \mathrm{min}$. (b) Rietveld refinement of high-resolution synchrotron XRD patterns at 290, 200, and $100 \mathrm{~K}$. Insets show the enlarged patterns corresponding to the peaks of the orthorhombic phase. The red lines and overlying crosses show the calculated and observed intensities, respectively. The pink and green lines indicate the positions of the calculated Bragg reflections of orthorhombic and cubic $\mathrm{CsPCl}_{3}$, respectively. The difference between the observed and calculated profiles is plotted in blue. (c, d) Percentages (c) and cell volumes (d) of the cubic and orthorhombic phases in high-quality $\mathrm{CsPbCl}_{3} \mathrm{NCs}$ at different temperatures.

temperatures by using a two-phase model (Figure 3b, Figures S14 and S15, and Tables S10-S14). As shown in Figure 3c, the ratio of the orthorhombic polymorph in NCs at RT is $48.4 \%$, which increases upon cooling, corresponding to a ratio of $75.3 \%$ at $100 \mathrm{~K}$. This is in sharp contrast with the highly defective NCs, suggesting that a lower concentration of defects causes a greatly retarded conversion of the cubic polymorph to the orthorhombic. Beyond this, we also find that the highquality NCs show a smaller variation in the volume of the unit cell for both the cubic and orthorhombic phases than the highly defective cousins (Figure $3 \mathrm{~d}$ ), meaning that lowerconcentration defects favor resistance of lattice contraction upon cooling (i.e., lower-concentration defects result in a robust lattice). Collectively, our results unambiguously indicate that, similar to the hybrid cousins, ${ }^{19}$ the phase transition behavior of $\mathrm{CsPCl}_{3} \mathrm{NCs}$ is defect-correlated and that the concentration of certain defects (e.g., $\mathrm{Cl}$ vacancies and/or $\mathrm{Cl}$ - vacancy-related defect complexes) not only govern the RT structure but also the phase transition behavior over an extremely large temperature interval (100-290 K).

Our experimental measurements reveal that the low PLQY of $\mathrm{CsPbCl}_{3} \mathrm{NCs}$ at $\mathrm{RT}$ can be attributed to the nonradiative trap centers induced by the $\mathrm{Cl}$ vacancies. To gain deep insights into the crystal structure and vacancy-related phase transition behavior as observed in $\mathrm{CsPbCl}_{3} \mathrm{NCs}$, we performed the CarParrinello molecular dynamics (CPMD) calculations on the 3 $\times 3 \times 3 \mathrm{CsPbCl}_{3}$ supercells without and with a $\mathrm{Cl}$ vacancy (Figure 4). Overall, starting from experimental crystal structures, $\mathrm{CsPCl}_{3}$ experiences a strong thermal fluctuation and would not retain the perfect cubic phase after 2 ps. As illustrated in Figure $4 c$,d, in comparison with the perfect case, the $\mathrm{Pb}-\mathrm{Cl}-\mathrm{Pb}$ bonds in the defective crystal undergo a large fluctuation with an average tilting angle between $155^{\circ}$ and $167^{\circ}$ at $290 \mathrm{~K}$ because the $\mathrm{Cl}$ vacancy leads to a strong lattice 
a
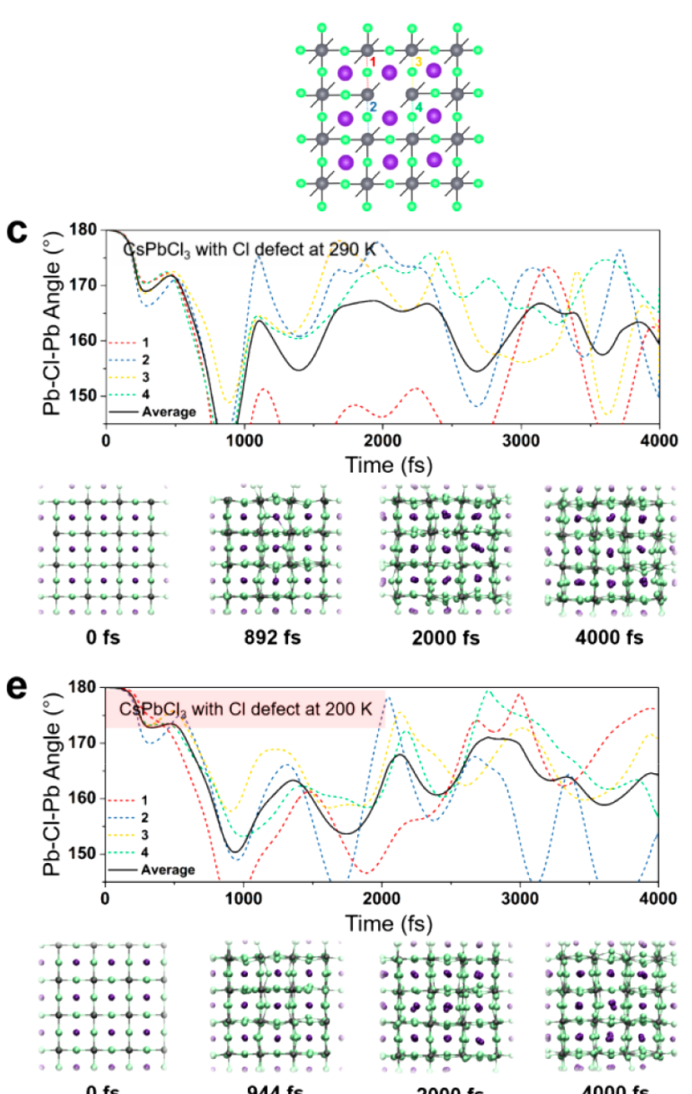

0 fs

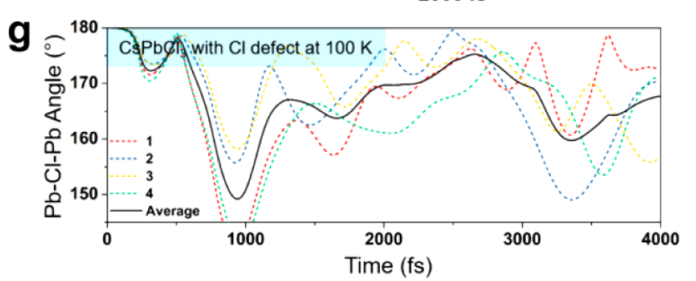

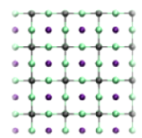

0 fs

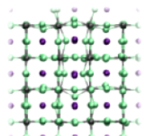

944 fs

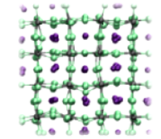

$2000 \mathrm{fs}$

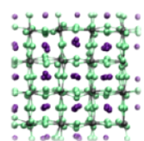

$4000 \mathrm{fs}$ b

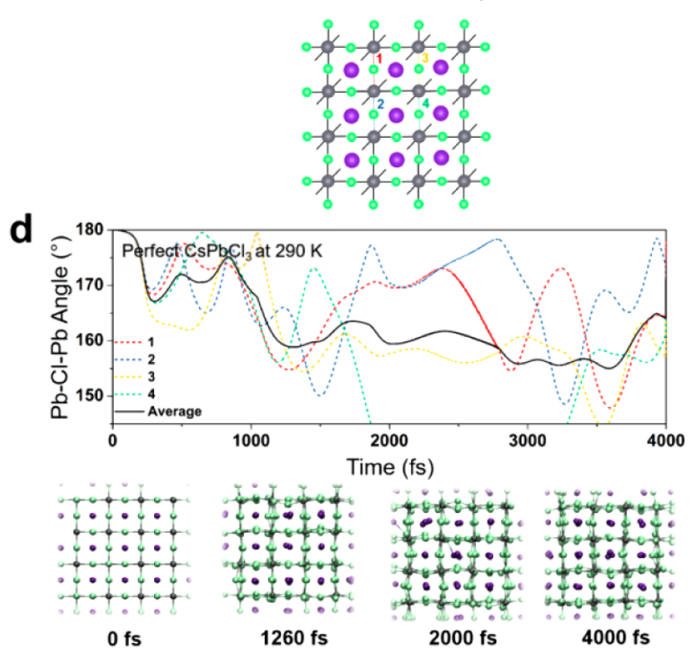

f
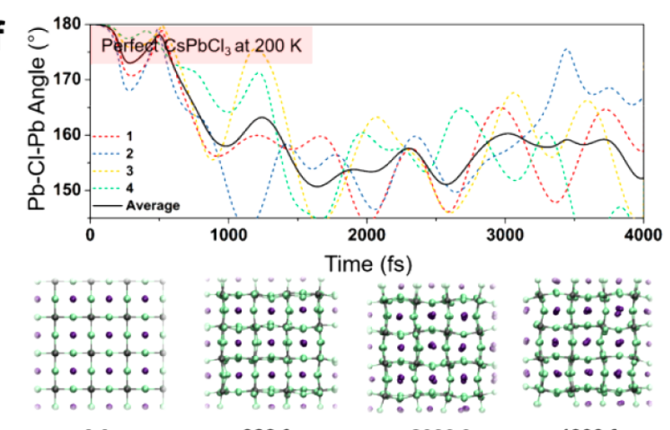

0 fs

h
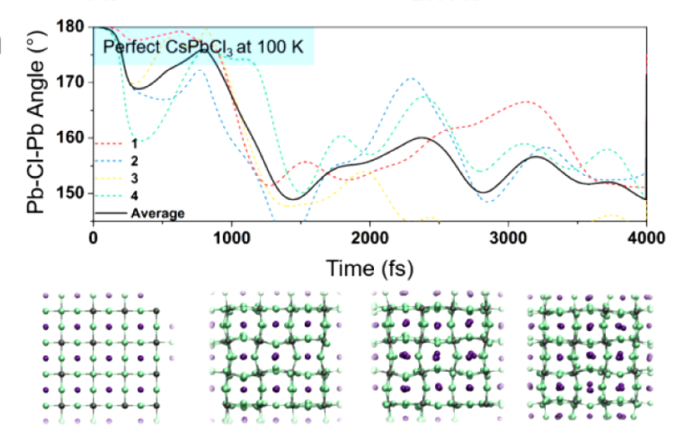

0 fs

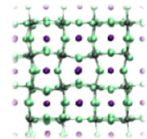

1396 fs

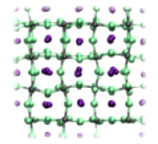

2000 fs

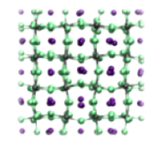

4000 fs

Figure 4. CPMD simulations. (a, b) Illustrations of the four $\mathrm{Pb}-\mathrm{Cl}-\mathrm{Pb}$ band angles taken from $3 \times 3 \times 3 \mathrm{CsPbCl}{ }_{3}$ supercells. The $\mathrm{Pb}-\mathrm{Cl}-$ $\mathrm{Pb}$ bond angle evolution starting from initial cubic-phase $\mathrm{CsPbCl}_{3}$ at $290 \mathrm{~K}(\mathrm{c}, \mathrm{d}), 200 \mathrm{~K}(\mathrm{e}, \mathrm{f})$, and $100 \mathrm{~K}$ (g, h) with and without $\mathrm{Cl}$ vacancy and the corresponding snapshots obtained from CPMD calculations.

distortion (i.e., octahedral titling) surrounding this vacancy. Such local structural deformation can release the lattice strain, resulting in less $\mathrm{Pb}-\mathrm{Cl}-\mathrm{Pb}$ tilting away from the $\mathrm{Cl}$ vacancy center (see the plots of $\mathrm{Pb}-\mathrm{Cl}-\mathrm{Pb}$ bond angles in Figure $\mathrm{S} 16$ ). Interestingly, reducing the temperature from 290 to $100 \mathrm{~K}$ inhibits the $\mathrm{Pb}-\mathrm{Cl}-\mathrm{Pb}$ tilting around the $\mathrm{Cl}$ vacancy of $\mathrm{CsPCl}_{3}$ supercell (Figure $4 \mathrm{c}, \mathrm{e}, \mathrm{g}$ ), but promotes the $\mathrm{Pb}-\mathrm{Cl}-$ $\mathrm{Pb}$ tilting away from the $\mathrm{Cl}$ vacancy, i.e., the average $\mathrm{Pb}-\mathrm{Cl}-$ $\mathrm{Pb}$ bond angle around a $\mathrm{Cl}$ vacancy increases (between $165^{\circ}$ and $175^{\circ}$ ) and it decreases away from a $\mathrm{Cl}$ vacancy (between $145^{\circ}$ and $155^{\circ}$, see Figure $\left.\mathrm{S} 16 \mathrm{c}, \mathrm{d}\right)$. This indicates the contraction of the resulting $\mathrm{CsPCl}_{3}$ crystal with a $\mathrm{Cl}$ vacancy at low temperatures, making it closer to the typical orthorhombic phase with the $\mathrm{Pb}-\mathrm{Cl}-\mathrm{Pb}$ tilting angle of $150-155^{\circ}$. In a sharp contrast, the perfect $\mathrm{CsPbCl}_{3}$ crystal inclines to keep the orthorhombic phase with the average $\mathrm{Pb}-$
$\mathrm{Cl}-\mathrm{Pb}$ bond angle of $\sim 157^{\circ}$, as no lattice relaxation occurs for the phase change (Figure $4 \mathrm{~d}, \mathrm{f}, \mathrm{h}$ ). It is worth noting that the high-quality $\mathrm{CsPCl}_{3} \mathrm{NCs}$ are not perfect but contain a small amount of $\mathrm{Cl}$ vacancies, which gives rise to the mixed cubic/ orthorhombic phase as observed in our experiments. Collectively, our CPMD calculations support that the $\mathrm{Cl}$ vacancy can trigger the phase change of $\mathrm{CsPbCl}_{3} \mathrm{NCs}$ : (i) the $\mathrm{Cl}$ vacancy induces a strong local lattice deformation at $\mathrm{RT}$ (that results in the occurrence of quasi-cubic phase way from the vacancy) in highly defective $\mathrm{CsPCl}_{3} \mathrm{NCs}$ as well as a large $\mathrm{Pb}-\mathrm{Cl}-\mathrm{Pb}$ tilting at low temperatures far from the vacancy (that results in the contraction of the lattice); (ii) for the highquality $\mathrm{CsPCl}_{3} \mathrm{NCs}$, the less $\mathrm{Pb}-\mathrm{Cl}-\mathrm{Pb}$ tilting releases lattice strain, which can suppress the phase transition from quasi-cubic to orthorhombic. We underscore that in the $\mathrm{CsPbCl}_{3} \mathrm{NCs}$, the appearance of other defects, such as the 
$\mathrm{Cs}^{+}-\mathrm{Cl}^{-}$vacancy complex, can further promote crystal lattice deformations and impact the phase transition, which is more complicated than our used model containing one $\mathrm{Cl}$ vacancy.

We next examined whether other $\mathrm{CsPbX}_{3} \mathrm{NCs}$ demonstrate a similar phenomenon as observed in $\mathrm{CsPbCl}_{3} \mathrm{NCs}$. For this purpose, we prepared defective and high-quality $\mathrm{Cs}_{\mathrm{PbBr}} \mathrm{NCs}$ with PLQYs of $43.8 \%$ and $97.0 \%$, respectively. The average size of both defective and high-quality $\mathrm{CsPBr}_{3} \mathrm{NCs}$ is $8.6 \mathrm{~nm}$ (Figure S17). It is also noted that both NCs are capped with the same class of ligands. These facts indicate that the ligand and NC's size have a negligible effect on the structure of $\mathrm{CsPbBr}{ }_{3} \mathrm{NCs}$, which is similar to the case of $\mathrm{CsPbCl}_{3} \mathrm{NCs}$. As shown in Figure S18, defective and high-quality $\mathrm{CsPbr}_{3} \mathrm{NCs}$ exhibit a reversible phase transition across a wide temperature interval. However, close examination of the diffraction patterns (e.g., those at $103 \mathrm{~K}$ ) obviously evidence that defects can also trigger the phase transition behavior of $\mathrm{CsPbBr}_{3} \mathrm{NCs}$.

In conclusion, we have revealed that structural defects dictate the structure and phase transition behaviors of allinorganic perovskite NCs across wide temperature intervals. We found that the cubic subdomains in highly defective

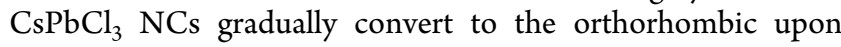
cooling the RT quasi-cubic NCs, while high-quality cousins exhibit a significantly retarded phase transition from RT mixed cubic and orthorhombic subdomains. Importantly, we found that the defect-triggered phase transition also exists in other allinorganic halide perovskite NCs. Our work highlights the intrinsic complexity of a seemingly simple $\mathrm{CsPbX}_{3}$ perovskite structure when downsizing and structural defects meet together and sheds new light on the important role of defects in impacting the structures of perovskite NCs.

\section{ASSOCIATED CONTENT}

\section{S Supporting Information}

The Supporting Information is available free of charge on the ACS Publications website at DOI: 10.1021/acsmaterialslett.9b00128.

Experimental methods, TEM images, additional XRD patterns, absorption and PL spectra, additional CPMD simulation results, and supplementary tables of Rietveld refinement results (PDF)

\section{AUTHOR INFORMATION}

\section{Corresponding Authors}

*E-mail: timothyhsun@gmail.com.

*E-mail: omar.abdelsaboor@kaust.edu.sa.

\section{ORCID $\odot$}

Ju-Ping Ma: 0000-0002-7436-9148

Jun Yin: 0000-0002-1749-1120

Osman M. Bakr: 0000-0002-3428-1002

Omar F. Mohammed: 0000-0001-8500-1130

Hong-Tao Sun: 0000-0002-0003-7941

\section{Author Contributions}

\#J.-P. Ma, J. Yin, Y.-M. Chen, and Q. Zhao contributed equally to this work.

\section{Notes}

The authors declare no competing financial interest.

\section{ACKNOWLEDGMENTS}

This work was supported by the National Natural Science Foundation of China (Grant Nos. 11874275 and 11574225) and two projects funded by the Priority Academic Program Development of Jiangsu Higher Education Institutions (PAPD) and the State Key Laboratory of Luminescence and Applications, Chinese Academy of Sciences. The SPring- 8 experiment was carried out with the approval of the Japan Synchrotron Radiation Research Institute (JASRI; Proposal No. 2017B1309). Computational work was supported by the Supercomputing Laboratory at King Abdullah University of Science and Technology (KAUST).

\section{REFERENCES}

(1) Saparov, B.; Mitzi, D. B. Organic-Inorganic Perovskites: Structural Versatility for Functional Materials Design. Chem. Rev. 2016, 116, 4558-4596.

(2) Stoumpos, C. C.; Kanatzidis, M. G. Halide Perovskites: Poor Man's High-Performance Semiconductors. Adv. Mater. 2016, 28, $5778-5793$

(3) Sutherland, B. R.; Sargent, E. H. Perovskite Photonic Sources. Nat. Photonics 2016, 10, 295-302.

(4) Correa-Baena, J.-P.; Saliba, M.; Buonassisi, T.; Grätzel, M.; Abate, A.; Tress, W.; Hagfeldt, A. Promises and Challenges of Perovskite Solar Cells. Science 2017, 358, 739-744.

(5) Chen, Z.; Turedi, B.; Alsalloum, A. Y.; Yang, C.; Zheng, X.; Gereige, I.; AlSaggaf, A.; Mohammed, O. F.; Bakr, O. M. SingleCrystal $\mathrm{MAPbI}_{3}$ Perovskite Solar Cells Exceeding 21\% Power Conversion Efficiency. ACS Energy Lett. 2019, 4, 1258-1259.

(6) El-Ballouli, A. O.; Bakr, O. M.; Mohammed, O. F. Compositional, Processing, and Interfacial Engineering of Nanocrystal- and Quantum-Dot-Based Perovskite Solar Cells. Chem. Mater. 2019, DOI: 10.1021 /acs.chemmater.9b01268.

(7) Noh, J. H.; Im, S. H.; Heo, J. H.; Mandal, T. N.; Seok, S. I. Chemical Management for Colorful, Efficient, and Stable InorganicOrganic Hybrid Nanostructured Solar Cells. Nano Lett. 2013, 13, 1764-1769.

(8) Jeon, N. J.; Noh, J. H.; Yang, W. S.; Kim, Y. C.; Ryu, S.; Seo, J.; Seok, S. I. Compositional Engineering of Perovskite Materials for High-Performance Solar Cells. Nature 2015, 517, 476-480.

(9) Akkerman, Q. A.; D'Innocenzo, V.; Accornero, S.; Scarpellini, A.; Petrozza, A.; Prato, M.; Manna, L. Tuning the Optical Properties of Cesium Lead Halide Perovskite Nanocrystals by Anion Exchange Reactions. J. Am. Chem. Soc. 2015, 137, 10276-10281.

(10) Abdi-Jalebi, M.; Andaji-Garmaroudi, Z.; Cacovich, S.; Stavrakas, C.; Philippe, B.; Richter, J. M.; Alsari, M.; Booker, E. P.; Hutter, E. M.; Pearson, A. J.; Lilliu, S.; Savenije, T. J.; Rensmo, H.; Divitini, G.; Ducati, C.; Friend, R. H.; Stranks, S. D. Maximizing and Stabilizing Luminescence from Halide Perovskites with Potassium Passivation. Nature 2018, 555, 497-501.

(11) Zhou, Y.; Chen, J.; Bakr, O. M.; Sun, H.-T. Metal-Doped Lead Halide Perovskites: Synthesis, Properties, and Optoelectronic Applications. Chem. Mater. 2018, 30, 6589-6613.

(12) Yin, J.; Ahmed, G. H.; Bakr, O. M.; Brédas, J.-L.; Mohammed, O. F. Unlocking the Effect of Trivalent Metal Doping in All-Inorganic $\mathrm{CsPbBr}_{3}$ Perovskite. ACS Energy Lett. 2019, 4, 789-795.

(13) Fang, H.-H.; Raissa, R.; Abdu-Aguye, M.; Adjokatse, S.; Blake, G. R.; Even, J.; Loi, M. A. Photophysics of Organic-Inorganic Hybrid Lead Iodide Perovskite Single Crystals. Adv. Funct. Mater. 2015, 25, 2378-2385.

(14) Weller, M. T.; Weber, O. J.; Henry, P. F.; Di Pumpo, A. M.; Hansen, T. C. Complete Structure and Cation Orientation in the Perovskite Photovoltaic Methylammonium Lead Iodide between 100 and 352 K. Chem. Commun. 2015, 51, 4180-4183.

(15) Dar, M. I.; Jacopin, G.; Meloni, S.; Mattoni, A.; Arora, N.; Boziki, A.; Zakeeruddin, S. M.; Rothlisberger, U.; Grätzel, M. Origin of Unusual Bandgap Shift and Dual Emission in Organic-Inorganic Lead Halide Perovskites. Sci. Adv. 2016, 2, No. e1601156.

(16) Fabini, D. H.; Stoumpos, C. C.; Laurita, G.; Kaltzoglou, A.; Kontos, A. G.; Falaras, P.; Kanatzidis, M. G.; Seshadri, R Reentrant Structural and Optical Properties and Large Positive Thermal 
Expansion in Perovskite Formamidinium Lead Iodide. Angew. Chem., Int. Ed. 2016, 55, 15392-15396.

(17) Page, K.; Siewenie, J. E.; Quadrelli, P.; Malavasi, L. Short-Range Order of Methylammonium and Persistence of Distortion at the Local Scale in $\mathrm{MAPbBr}_{3}$ Hybrid Perovskite. Angew. Chem., Int. Ed. 2016, 55, 14320-14324.

(18) Bernasconi, A.; Malavasi, L. Direct Evidence of Permanent Octahedra Distortion in $\mathrm{MAPbBr}_{3}$ Hybrid Perovskite. ACS Energy Lett. 2017, 2, 863-868.

(19) Dobrovolsky, A.; Merdasa, A.; Unger, E. L.; Yartsev, A.; Scheblykin, I. G. Defect-Induced Local Variation of Crystal Phase Transition Temperature in Metal-Halide Perovskites. Nat. Commun. 2017, 8, 34.

(20) Yaffe, O.; Guo, Y.; Tan, L. Z.; Egger, D. A.; Hull, T.; Stoumpos, C. C.; Zheng, F.; Heinz, T. F.; Kronik, L.; Kanatzidis, M. G.; Owen, J. S.; Rappe, A. M.; Pimenta, M. A.; Brus, L. E. Local Polar Fluctuations in Lead Halide Perovskite Crystals. Phys. Rev. Lett. 2017, 118, 136001.

(21) Neutzner, S.; Srimath Kandada, A. R.; Lanzani, G.; Petrozza, A. A Dual-Phase Architecture for Efficient Amplified Spontaneous Emission in Lead Iodide Perovskites. J. Mater. Chem. C 2016, 4, 4630-4633.

(22) Jia, Y.; Kerner, R. A.; Grede, A. J.; Rand, B. P.; Giebink, N. C. Continuous-Wave Lasing in an Organic-Inorganic Lead Halide Perovskite Semiconductor. Nat. Photonics 2017, 11, 784-788.

(23) Protesescu, L.; Yakunin, S.; Bodnarchuk, M. I.; Krieg, F.; Caputo, R.; Hendon, C. H.; Yang, R. X.; Walsh, A.; Kovalenko, M. V. Nanocrystals of Cesium Lead Halide Perovskites $\left(\mathrm{CsPbX}_{3}, \mathrm{X}=\mathrm{Cl}, \mathrm{Br}\right.$, and I): Novel Optoelectronic Materials Showing Bright Emission with Wide Color Gamut. Nano Lett. 2015, 15, 3692-3696.

(24) Yakunin, S.; Protesescu, L.; Krieg, F.; Bodnarchuk, M. I.; Nedelcu, G.; Humer, M.; De Luca, G.; Fiebig, M.; Heiss, W.; Kovalenko, M. V. Low-Threshold Amplified Spontaneous Emission and Lasing from Colloidal Nanocrystals of Caesium Lead Halide Perovskites. Nat. Commun. 2015, 6, 8056.

(25) Dutta, A.; Behera, R. K.; Pal, P.; Baitalik, S.; Pradhan, N. NearUnity Photoluminescence Quantum Efficiency for All $\mathrm{CsPbX}$ (X=Cl, $\mathrm{Br}$, and I) Perovskite Nanocrystals: a Generic Synthesis Approach. Angew. Chem. 2019, 131, 5608-5612.

(26) Ahmed, T.; Seth, S.; Samanta, A. Boosting the Photoluminescence of $\mathrm{CsPbX}_{3}(\mathrm{X}=\mathrm{Cl}, \mathrm{Br}, \mathrm{I})$ Perovskite Nanocrystals Covering a Wide Wavelength Range by Postsynthetic Treatment with Tetrafluoroborate Salts. Chem. Mater. 2018, 30, 3633-3637.

(27) Tong, Y.; Bladt, E.; Ayguler, M. F.; Manzi, A.; Milowska, K. Z.; Hintermayr, V. A.; Docampo, P.; Bals, S.; Urban, A. S.; Polavarapu, L.; Feldmann, J. Highly Luminescent Cesium Lead Halide Perovskite Nanocrystals with Tunable Composition and Thickness by Ultrasonication. Angew. Chem., Int. Ed. 2016, 55, 13887-13892.

(28) Zhang, X.; Lin, H.; Huang, H.; Reckmeier, C.; Zhang, Y.; Choy, W. C.; Rogach, A. L. Enhancing the Brightness of Cesium Lead Halide Perovskite Nanocrystal Based Green Light-Emitting Devices through the Interface Engineering with Perfluorinated Ionomer. Nano Lett. 2016, 16, 1415-1420.

(29) Huang, H.; Bodnarchuk, M. I.; Kershaw, S. V.; Kovalenko, M. V.; Rogach, A. L. Lead Halide Perovskite Nanocrystals in the Research Spotlight: Stability and Defect Tolerance. ACS Energy Lett. 2017, 2, 2071-2083.

(30) Li, Z.; Kong, L.; Huang, S.; Li, L. Highly Luminescent and Ultrastable $\mathrm{CsPbBr}_{3}$ Perovskite Quantum Dots Incorporated into a Silica/Alumina Monolith. Angew. Chem., Int. Ed. 2017, 56, 81348138.

(31) Kovalenko, M. V.; Protesescu, L.; Bodnarchuk, M. I. Properties and Potential Optoelectronic Applications of Lead Halide Perovskite Nanocrystals. Science 2017, 358, 745-750.

(32) Imran, M.; Caligiuri, V.; Wang, M.; Goldoni, L.; Prato, M.; Krahne, R.; De Trizio, L.; Manna, L. Benzoyl Halides as Alternative Precursors for the Colloidal Synthesis of Lead Based Halide Perovskite Nanocrystals. J. Am. Chem. Soc. 2018, 140, 2656-2664.

(33) Muduli, S.; Pandey, P.; Devatha, G.; Babar, R.; Thripuranthaka, M.; Kothari, D. C.; Kabir, M.; Pillai, P.; Ogale, S. Photoluminescence
Quenching in Self-Assembled $\mathrm{CsPBr}_{3}$ Quantum Dots on Few- Layer Black Phosphorous Sheets. Angew. Chem. 2018, 130, 7808-7812.

(34) Cohen, M. I.; Young, K. F.; Chang, T. T.; Brower, W. S. Phase Transitions in $\mathrm{CsPbCl}_{3}$. J. Appl. Phys. 1971, 42, 5267-5272.

(35) Ohta, H.; Harada, J.; Hirotsu, S. Superstructure and Phase Transitions in $\mathrm{CsPbCl}_{3}$. Solid State Commun. 1973, 13, 1969-1972.

(36) Hirotsu, S.; Harada, J.; Iizumi, M.; Gesi, K. Structural Phase Transitions in CsPbBr ${ }_{3}$. J. Phys. Soc. Jpn. 1974, 37, 1393-1398.

(37) Fujii, Y.; Hoshino, S.; Yamada, Y.; Shirane, G. NeutronScattering Study on Phase Transitions of $\mathrm{CsPbCl}_{3}$. Phys. Rev. B 1974, 9, 4549-4559.

(38) Hirotsu, S.; Suzuki, T. Critical Thermodynamic Properties around the Successive Phase Transitions of $\mathrm{CsPbBr}_{3}$ and $\mathrm{CsPbCl}_{3}$. Ferroelectrics 1978, 20, 179-180.

(39) Hidaka, M.; Okamoto, Y.; Zikumaru, Y. Structural Phase Transition of $\mathrm{CsPbCl}_{3}$ below Room Temperature. Phys. stat. sol.(a) 1983, 79, 263-269.

(40) Bertolotti, F.; Protesescu, L.; Kovalenko, M. V.; Yakunin, S.; Cervellino, A.; Billinge, S. J. L.; Terban, M. W.; Pedersen, J. S.; Masciocchi, N.; Guagliardi, A. Coherent Nanotwins and Dynamic Disorder in Cesium Lead Halide Perovskite Nanocrystals. ACS Nano 2017, 11, 3819-3831.

(41) Cottingham, P.; Brutchey, R. L. Depressed Phase Transitions and Thermally Persistent Local Distortions in $\mathrm{CsPbBr}_{3}$ Quantum Dots. Chem. Mater. 2018, 30, 6711-6716.

(42) Kirschner, M. S.; Diroll, B. T.; Guo, P.; Harvey, S. M.; Helweh, W.; Flanders, N. C.; Brumberg, A.; Watkins, N. E.; Leonard, A. A.; Evans, A. M.; Wasielewski, M. R.; Dichtel, W. R.; Zhang, X.; Chen, L. X.; Schaller, R. D. Photoinduced, Reversible Phase Transitions in AllInorganic Perovskite Nanocrystals. Nat. Commun. 2019, 10, 504.

(43) Tenailleau, C.; Aharon, S.; Cohen, B.-E.; Etgar, L. Cell Refinement of $\mathrm{CsPbBr}_{3}$ Perovskite Nanoparticles and Thin Films. Nanoscale Adv. 2019, 1, 147-153.

(44) Saidaminov, M. I.; Kim, J.; Jain, A.; Quintero-Bermudez, R.; Tan, H.; Long, G.; Tan, F.; Johnston, A.; Zhao, Y.; Voznyy, O.; Sargent, E. H. Suppression of Atomic Vacancies Via Incorporation of Isovalent Small Ions to Increase the Stability of Halide Perovskite Solar Cells in Ambient Air. Nature Energy 2018, 3, 648-654.

(45) Yong, Z. J.; Guo, S. Q.; Ma, J. P.; Zhang, J. Y.; Li, Z. Y.; Chen, Y. M.; Zhang, B. B.; Zhou, Y.; Shu, J.; Gu, J. L.; Zheng, L. R.; Bakr, O. M.; Sun, H. T. Doping-Enhanced Short-Range Order of Perovskite Nanocrystals for Near-Unity Violet Luminescence Quantum Yield. J. Am. Chem. Soc. 2018, 140, 9942-9951.

(46) Becker, M. A.; Vaxenburg, R.; Nedelcu, G.; Sercel, P. C.; Shabaev, A.; Mehl, M. J.; Michopoulos, J. G.; Lambrakos, S. G.; Bernstein, N.; Lyons, J. L.; StÖferle, T.; Mahrt, R. F.; Kovalenko, M. V.; Norris, D. J.; Rainò, G.; Efros, A. L. Bright Triplet Excitons in Caesium Lead Halide Perovskites. Nature 2018, 553, 189-193.

(47) Diroll, B. T.; Zhou, H.; Schaller, R. D. Low-Temperature Absorption, Photoluminescence, and Lifetime of $\mathrm{CsPbX}_{3}(\mathrm{X}=\mathrm{Cl}, \mathrm{Br}$, I). Adv. Funct. Mater. 2018, 28, 1800945.

(48) Ahmed, G. H.; El-Demellawi, J. K.; Yin, J.; Pan, J.; Velusamy, D. B.; Hedhili, M. N.; Alarousu, E.; Bakr, O. M.; Alshareef, H. N.; Mohammed, O. F. Giant Photoluminescence Enhancement in $\mathrm{CsPbCl}_{3}$ Perovskite Nanocrystals by Simultaneous Dual-Surface Passivation. ACS Energy Lett. 2018, 3, 2301-2307.

(49) Kawaguchi, S.; Takemoto, M.; Osaka, K.; Nishibori, E.; Moriyoshi, C.; Kubota, Y.; Kuroiwa, Y.; Sugimoto, K. HighThroughput Powder Diffraction Measurement System Consisting of Multiple MYTHEN Detectors at Beamline BL02B2 of SPring-8. Rev. Sci. Instrum. 2017, 88, 085111. 